\title{
New Drugs Approval
}

\section{Lenvima}

Generic Name: lenvatinib

Date of Approval: February 13, 2015

Company: Eisai Co., Ltd.

\section{Treatment for: Thyroid Cancer}

The U.S. Food and Drug Administration (FDA) has approved Lenvima (lenvatinib), an oral kinase inhibitor used to treat patients with progressive, differentiated thyroid cancer (DTC) whose disease progressed despite receiving radioactive iodine therapy (radioactive iodine refractory disease).

Lenvima is a targeted therapy that fights thyroid cancer by deterring the growth of new blood vessels that could help feed the cancer, researchers said. It delayed progression of advanced thyroid cancer by 18 months, compared with four months for patients treated with a placebo, the trial found. Results of the study, which was funded by drug manufacturer Eisai, were published in the Feb. 12 issue of the New England Journal of Medicine.

\section{Dutrebis}

Generic name: lamivudine and raltegravir

Date of approved: February 6th, 2015

Company: Merck

\section{Treatment for: HIV Infection}

Dutrebis (lamivudine and raltegravir) is a fixed-dose combination of a nucleoside analog reverse transcriptase inhibitor and an integrase strand transfer inhibitor for the treatment of HIV-1 infection. Dutrebis will NOT be made commercially available in the U.S. at this time.

\section{Ibrance}

Generic Name: palbociclib

Date of Approval: February 3, 2015

Company: Pfizer Inc.

\section{Treatment for: Breast Cancer}

Ibrance is the first approved Cyclin-Dependent Kinase 4/6 (CDK 4/6) Inhibitor. The U.S. Food and Drug Administration (FDA) has granted accelerated approval of
Ibrance (palbociclib), an oral inhibitor of cyclin-dependent kinases (CDKs) 4 and 6, for the combination treatment of postmenopausal women with estrogen receptor-positive, human epidermal growth factor receptor 2-negative (ER+/HER2-) advanced breast cancer.

Ibrance is to be used in combination with letrozole, another drug used to treat certain kinds of breast cancer in postmenopausal women, the agency said.

The FDA's approval of Ibrance was based on a study that included 165 postmenopausal women who had ERpositive, HER2-negative advanced breast cancer and had not been treated for their advanced disease. Those who received Ibrance plus letrozole lived about 20 months without their disease progressing, compared to about 10 months for those who took only letrozole. Data on overall survival rates is not yet available.

Common side effects of Ibrance include low white and red blood cell counts, fatigue, nausea, upper respiratory infection, diarrhea, vomiting, hair loss, inflammation of the lining of the mouth, decreased appetite, nosebleeds, and damage to the nerves in the extremities, the agency said.

\section{Glyxambi}

Generic Name: empagliflozin and linagliptin

Date of Approval: January 30, 2015

Company: Boehringer Ingelheim Pharmaceuticals, Inc.

\section{Treatment for: Type 2 Diabetes}

Glyxambi is the first prescription medication in U.S. to combine the dual mechanisms of action of SGLT2 and DPP-4 inhibitors. The U.S. Food and Drug Administration (FDA) has approved Glyxambi (empagliflozin/linagliptin), a once-daily sodium glucose co-transporter-2 (SGLT2) inhibitor and dipeptidyl peptidase-4 (DPP-4) inhibitor fixed-dose combination for the treatment of adults with type 2 diabetes.

\section{Olopatadine}

Generic Name: olopatadine ophthalmic (OH loe PAT a deen) Brand Name: Pataday, Patanol 


\section{Treatment for: symptoms of allergic conditions in eyes}

Olopatadine is an antihistamine that reduces the natural chemical histamine in the body. Histamine can produce symptoms of itching or watery eyes.

Olopatadine ophthalmic is used to treat ocular (eye) symptoms of allergic conditions, such as inflammation, itching, watering, and burning. Olopatadine ophthalmic may also be used for other purposes not listed in this medication guide.

\section{Prezcobix}

Generic Name: darunavir and cobicistat Date of Approval: January 29, 2015

Company: Janssen Pharmaceuticals, Inc.

\section{Treatment for: $H I V-1$ Infection}

Prezcobix joins Evotaz as the first approved protease inhibitor and cobicistat fixed-dose combinations. The U.S. Food and Drug Administration (FDA) has approved Prezcobix, a once-daily, fixed-dose combination containing the protease inhibitor darunavir (marketed as Prezista), and the pharmacokinetic enhancing CYP3A4 inhibitor cobicistat (marketed as Tybost) for the treatment of human immunodeficiency virus (HIV-1) infection.

\section{Evotaz}

Generic Name: atazanavir and cobicistat Date of Approval: January 29, 2015

Company: Bristol-Myers Squibb Company

\section{Treatment for: HIV-1 Infection}

Evotaz joins Prezcobix as the first approved protease inhibitor and cobicistat fixed-dose combinations. The U.S. Food and Drug Administration (FDA) has approved Evotaz (atazanavir $300 \mathrm{mg}$ and cobicistat $150 \mathrm{mg}$ ), a protease inhibitor and pharmacokinetic enhancer fixeddose combination for the treatment of HIV-1 infection in adults. Evotaz is a once-daily tablet combining atazanavir, which is marketed as Reyataz, and cobicistat, which is marketed as Tybost by Gilead Sciences, Inc.

\section{Sources: www.drugs.com; www.worldpharmanews.com}

Information collected and compiled by:

\section{Mr. Md. Akbar Hossain}

Assistant Professor, Department of Pharmacy

Dhaka International University

\section{Natpara}

Generic Name: parathyroid hormone

Date of Approval: January 23, 2015

Company: NPS Pharmaceuticals, Inc.

\section{Treatment for: Hypocalcemia in Patients with} Hypoparathyroidism

Natpara is the first FDA-approved parathyroid hormone for hypoparathyroidism.

The U.S. Food and Drug Administration (FDA) has approved Natpara (parathyroid hormone), a bioengineered replica of human parathyroid hormone, as an adjunct to calcium and vitamin $\mathrm{D}$ to control hypocalcemia in patients with hypoparathyroidism.

\section{FDA Approves Lucentis as First Drug for Diabetic Retinopathy}

February 6, 2015 -- The U.S. Food and Drug Administration on Friday approved the drug Lucentis (ranibizumab) to treat diabetic retinopathy in patients with diabetic macular edema.

A leading cause of blindness among adults in the United States, diabetic retinopathy is the most common diabetic eye disease, the agency said in a news release.

In 2008,33 percent of adults with diabetes aged 40 or older had some form of diabetic retinopathy. In some patients who have diabetic retinopathy with diabetic macular edema, abnormal new blood vessels grow on the surface of the retina and if the vessels burst severe vision loss or blindness can occur.

Lucentis must be injected into the eye once a month by a doctor, and should be used alongside treatments to control blood sugar, blood pressure and cholesterol, according to the FDA. The drug works by inhibiting leakage of blood vessels and deterring the growth of blood vessels into the retina of the eye, said Dr. Pravin Dugel, a retinal specialist at Retinal Consultants of Arizona and an investigator in the medication's two clinical trials. 